\title{
Efficacy of intra-meibomian gland injection of the anti-VEGF agent bevacizumab for the treatment of meibomian gland dysfunction with lid-margin
} vascularity

This article was published in the following Dove Press journal:

Drug Design, Development and Therapy

\section{Xiaodan Jiang* \\ Yuexin Wang* \\ Huibin Lv \\ Yan Liu \\ Mingzhou Zhang \\ Xuemin Li}

Department of Ophthalmology, Peking University Third Hospital, Beijing, China

*These authors contributed equally to this work
Correspondence: Xuemin Li

Department of Ophthalmology,

Peking University Third Hospital,

49 Huayuan North Road, Haidian Qu,

Beijing 100083, China

Tel +86 I39 I I 254862

Email lxmlxm66@sina.com
Purpose: To investigate the efficacy of a novel treatment - intra-meibomian gland (MG) injection of the anti-VEGF agent bevacizumab - for MG dysfunction (MGD) with eyelid-margin vascularity.

Methods: A total of 26 eyes from 13 patients diagnosed with MGD and eyelid-margin vascularity were included in our study. Patients received intra-meibomian gland injections of bevacizumab $(150 \mu \mathrm{L}, 2.5 \mathrm{mg} / 0.1 \mathrm{~mL})$ at multiple sites with a $29 \mathrm{G}$ needle where telangiectasia was severe. The Ocular Surface Disease Index (OSDI), tear film, tear-breakup time (TBUT), eyelid-margin features, MG features, conjunctiva, and corneal staining were assessed at 1 day before injection and 1 week, 1 month, and 3 months after injection. Blood pressure, best-corrected visual acuity, intraocular pressure, and slit lamp examinations were performed to assure the safety of patients at 1 day before and 1 day, 1 week, 1 month, and 3 months after injection.

Results: Lid-margin vascularity, conjunctival injection, expressed secretion quality, expressivity of the MG, TBUT, corneal staining, and OSDI were significantly improved 1 week, 1 month, and 3 months after injection compared to baseline values. Lid-margin vascularity, conjunctival injection, meibomian gland expressivity, TBUT, and OSDI continued to improve; the greatest improvements were observed at 1 month and sustained for 3 months. Spearman's correlation analysis indicated that age and sex significantly influenced TBUT improvement. Females and older patients tended to have shorter baseline TBUT that followed a different trend from that of males and younger patients during postinjection visits, revealed by subgroup analysis. No local or systemic side effects were observed at follow-up visits.

Conclusion: This study is the first to explore a novel therapy for MGD - intra-MG injection of the anti-VEGF agent bevacizumab - and it demonstrates that the treatment is effective and safe in eliminating eyelid-margin vascularity, improving MG function and relieving clinical signs and symptoms of MGD.

Keywords: meibomian gland dysfunction, anti-VEGF, lid-margin vascularity

\section{Introduction}

Dry-eye disease (DED) is a common multifactorial ocular problem associated with ocular discomfort, blurred vision, tear-film instability, and even ocular surface damage. ${ }^{1,2}$ There are two main types of DE: aqueous-deficient and evaporative. ${ }^{3}$ Although both types of DE can occur in a patient separately or synergistically, epidemiological research has revealed that evaporative DE is more common. ${ }^{4,5}$ Meibomian gland dysfunction (MGD) is the predominant cause of evaporative DE. ${ }^{6,7}$ 
MGD affects both the MG and its adjacent lid structure. ${ }^{8}$ Rounding, notching, and hyperkeratosis of the eyelid margin, duct obstruction, and disuse atrophy of meibomian acini are commonly observed pathological events in MGD. ${ }^{9-11}$ Posterior lid-margin hyperemia with telangiectasia and increased vascularity is another pathological feature that is observed in $>60 \%$ of symptomatic MGD patients. ${ }^{12}$ Vascularization of the lid margin is more commonly seen in older patients, which possibly indicates the accumulation of exposure to various irritants. These pathological events are associated with a combination of multiple mechanisms, including inflammation, microbial factors, and lipid deficiency. ${ }^{13-15}$ These mechanisms are interdependent and form a vicious cycle that eventually causes DED through MG changes and tear-film instability. Tear-film imbalance leads to hyperosmolarity of the epithelium surface, which causes cell damage and apoptosis, and the induced inflammation arising from cell death further exacerbates instability of the tear film and DED symptoms. ${ }^{13}$

VEGF is the main factor regulating angiogenesis in multiple physiological processes, such as wound healing, tumor growth, and inflammation. ${ }^{16,17}$ Research has demonstrated that VEGF acts as a proinflammatory factor to stimulate the release of inflammatory factors, such as IL6, IL8, and TNF $\alpha .{ }^{18}$ Additionally, research has reported that an increase in VEGF was observed in the tear film of MGD patients compared to controls. ${ }^{19}$ Therefore, VEGF may be an intermittent factor between MGD and DED and may even promote the pathological events of MGD. Bevacizumab (Avastin; Genentech, San Francisco, CA, USA) is a humanized monoclonal antibody that binds and inhibits the biological activity of all VEGF subtypes. ${ }^{20}$ We have demonstrated that subconjunctival injection of bevacizumab is safe and effective in treating ocular surface inflammation in DED. ${ }^{21}$ This study explores an innovative treatment for MGD - intra-MG injection of the antivascularization agent bevacizumab - and assessed whether it is safe and effective in relieving the symptoms of MGD with lid-margin vascularity. ${ }^{20}$

\section{Methods}

\section{Patients}

This study was conducted according to the principles of the Declaration of Helsinki and was approved by the Human Research and Ethics Committee of Peking University Third Hospital. Written informed consent was obtained from each participant before enrollment. This was a prospective and open-label study. Subjects were recruited from the outpatient department of the Department of Ophthalmology of
Peking University Third Hospital between October 2016 and April 2017.

Inclusion criteria for this study were age 18-80 years; chief complaint of more than one of dryness, foreign-body sensation, burning, and tearing for $>6$ months; diagnosis of MGD with palpebral telangiectasia in both eyes; ${ }^{8}$ and willingness to cooperate with doctors in follow-up visits. Exclusion criteria were ocular structural abnormalities; history of ocular trauma or surgery; use of any treatment for DE or MGD, other than artificial tears, within the past month; active allergy, infection, or inflammatory disease at the ocular surface unrelated to DE or MGD; lacrimal drainage-system anomalies; contact-lens wear within the past month; systemic disease affecting the ocular surface; use of any systemic anti-inflammatory medicine; unstable systemic disease, such as hypertension or diabetes; and history of bevacizumab contraindications, such as heart failure, gastrointestinal perforation, or pregnancy. We certify that all applicable institutional and government regulations concerning the ethical use of human volunteers were followed during this research.

\section{Intra-meibomian gland injection}

In the operating room, participants received an intra-MG injection of bevacizumab $(150 \mu \mathrm{L}, 2.5 \mathrm{mg} / 0.1 \mathrm{~mL})$ with a 29 G needle (1 mL; BD, Franklin Lakes, NJ, USA). Iodine sterilization was applied first, after which iodine was wiped off with saline. Before injection, a drop of esthetic ropivacaine hydrochloride (Benoxil; Santen Pharmaceutical, Osaka, Japan) was applied to the conjunctival sac. Intra-MG injections entered from the MG orifice or the skin side near the orifice, then inserted into the main duct or around the main duct. Bevacizumab $(150 \mu \mathrm{L})$ was injected at multiple sites in the MGs in both upper and lower eyelids in regions of severe telangiectasia. Exact site numbers were different for different patients, normally three to five sites per eyelid $(50-100 \mu \mathrm{L}$ per eyelid); however, the total amount was the same per eye $(150 \mu \mathrm{L})$. One drop of levofloxacin (Ofloxacin; Santen Pharmaceutical) was applied to the eye after the injection. All injections were performed by the same doctor (XDJ).

\section{Clinical evaluation}

Subjects were evaluated five times during the study: 1 day before injection and 1 day, 1 week, 1 month, and 3 months after injection. Clinical assessments were performed in the following order: Ocular Surface Disease Index (OSDI); blood pressure; best-corrected visual acuity; intraocular pressure (IOP); tear-film assessment, including tear debris, tear foam, 
tear-meniscus height, and tear-breakup time (TBUT); grading of eyelid-margin features, such as rounding of the posterior margin, irregularity/notching of margin, vascularity of lid margin/telangiectasia, hyperkeratosis, lash loss, and anterior blepharitis; MG assessment, including the lid-orifice number present within the central $1 \mathrm{~cm}$, expressed secretion quality, and expressivity of the MG; and conjunctiva assessment, including conjunctival injection, conjunctivochalasis, and corneal staining. At the first follow-up visit 1 day after injection, only blood pressure, best-corrected visual acuity, IOP, and slit-lamp examination were required to ensure the safety of patients. Images of eyelid margin and corneal staining were obtained using an IM 900 slit lamp (IM 900; Köniz, Switzerland). An interval of 5 minutes was required between the different tests. All data were collected by two doctors (YXW and XDJ), and the average was defined as the final result.

\section{Symptom evaluation}

The OSDI (Allergan, Irvine, CA, USA) is a commonly applied questionnaire that evaluates the severity of DE symptoms with 12 questions. The OSDI is calculated as the sum of scores of all answered questions $\times 25$ divided by the total number of answered questions. OSDI scores range from 0 to 100 . OSDI scores define the ocular surface as normal (0-12) or having mild (13-22), moderate (23-32), or severe $\operatorname{DE}(>33) .^{22}$

\section{TBUT and corneal staining}

A $5 \mu \mathrm{L}$ volume of $2 \%$ sodium fluorescein was gently instilled into the patient's conjunctival sac using a micropipette to prevent reflex tearing. Next, the patient blinked several times to coat the ocular surface evenly with fluorescein. The examination was conducted under cobalt blue light using the IM 900. The patient was required to gaze steadily ahead without blinking. A stopwatch recorded the time that elapsed from the last complete blink to the first appearance of a dry spot. This procedure was performed three times to obtain the average value. For corneal staining evaluation, the cornea was divided into four sectors, ${ }^{23}$ and each sector was graded $0-3$ : $0=$ no staining, $1=$ punctate/stipple staining, $2=$ ball and linear staining, and $3=$ coalesced staining. ${ }^{24}$ These tests were evaluated by two doctors (XDJ and YXW) separately, and the average value was defined as the final result.

\section{Eyelid-margin features and gland- expression grading related to MGD}

Clinical features and gland-expression grading were performed as per Tomlinson et al. ${ }^{8}$ Eyelid features evaluated were rounding of posterior margin, irregularity/notching of margin, vascularity of lid margin/telangiectasia, hyperkeratosis, lash loss, and anterior blepharitis. These features were graded on a binary scale, where $0=$ no/normal and $1=$ yes $/$ abnormal. MG assessments included lid-orifice number present within central $1 \mathrm{~cm}$, expressed secretion quality into $4^{\circ}(0=$ clear, $1=$ cloudy, $2=$ granular, or $3=$ toothpaste $)$, and expressivity of the MG ( 1 = light, 2 = moderate, or $3=$ heavy pressure).

\section{Conjunctiva and tear-film assessment}

Conjunctival injection was graded according to the Institute for Eye Research scale: ${ }^{25}$ grade 0 (no hyperemia), grade 1 (mild focal hyperemia), grade 2 (mild diffused hyperemia), grade 3 (significant hyperemia), and grade 4 (severe hyperemia involving sclera). Conjunctivochalasis was graded as 0 (no persistent folds), 1 (one small fold), 2 (two or more folds not higher than the tear meniscus), and 3 (multiple folds higher than the tear meniscus). ${ }^{26}$ Tear debris and tear foam were assessed on a binary scale, with $0=$ no/normal and $1=$ yes/abnormal. The central upper and lower tear-meniscus heights were measured using slit-lamp microscopy (with a graticule in $0.05 \mathrm{~mm}$ units). ${ }^{27}$ Three consecutive readings were performed by two doctors (XDJ and YXW), and the average value was defined as the final result.

\section{Data analysis}

The Kolmogorov-Smirnov test was used to check the normality of the data distribution. Statistical analysis was performed using SPSS version 23.0. Comparisons between the data from the first visit and follow-up visits were performed using the McNemar $\chi^{2}$ test for nominal variables, Wilcoxon nonparametric test for ordinal variables, and repeated-measures analysis of variance (ANOVA) for continuous variables with a Bonferroni post hoc test.

In detail, the McNemar $\chi^{2}$ test was performed for rounding of posterior margin, irregularity/notching of margin, vascularity of lid margin/telangiectasia, hyperkeratosis, lash loss, anterior blepharitis, tear debris, and tear foam. Wilcoxon nonparametric tests were conducted for expressed secretion quality, expressivity of the MG, conjunctiva injection, conjunctivochalasis, and corneal staining. Repeated-measures ANOVAs were applied in the analysis of tear-meniscus height, lid-orifice number present within the central $1 \mathrm{~cm}$, TBUT, and the OSDI.

Additionally, differences in symptoms observed before and after injection at 1 week, 1 month, and 3 months were calculated, and Spearman's correlation analysis was performed 
to determine the effects of age and sex. Sex was recorded as $1=$ male and $2=$ female in the analysis. Age was divided into two categories: $1=<60$ years and $2=\geq 60$ years. Subgroup analyses were performed to determine differences in symptoms and signs between the sex and age subgroups, with $P<0.05$ indicating statistical significance.

\section{Results}

\section{Grading of MGD according to eyelid margins and gland expression}

A total of 26 eyes from 13 patients were included in the study. The average patient age was $57.15 \pm 12.84$ years, and the percentage of males was $30.8 \%$. Eyelid-margin features, MG expression, tear film, and conjunctiva were graded at baseline and 1 week, 1 month, and 3 months after bevacizumab injection. The results are shown in Figure 1 and summarized in Table 1.
As shown in Figure 2, lid-margin vascularity/telangiectasia was significantly improved at 1 week, 1 month, and 3 months after bevacizumab injection compared to baseline. The positive rate of lid-margin vascularity had decreased by approximately $30 \%$ by 1 week $(70.8 \%, P=0.003)$ and continued to improve, with the greatest decrease observed 1 month (33\%) after injection when compared with baseline and vascularity at 1 week $(P<0.0001, P=0.02)$. At 3 months postinjection, lid-margin vascularity sustained a relatively reduced rate when compared with baseline $(58.3 \%, P=0.002)$, as shown in Figure 1A. Similarly, when compared to baseline, a remarkable decrease in conjunctival injection was observed after bevacizumab injection, with the greatest reduction observed at 1 month, as shown in Figure $1 \mathrm{C}$.

Significantly improved MG function was observed post-injection, as indicated by lower grades of secretion quality and expressivity at 1 week $(1.71 \pm 0.13, P<0.0001$;
A

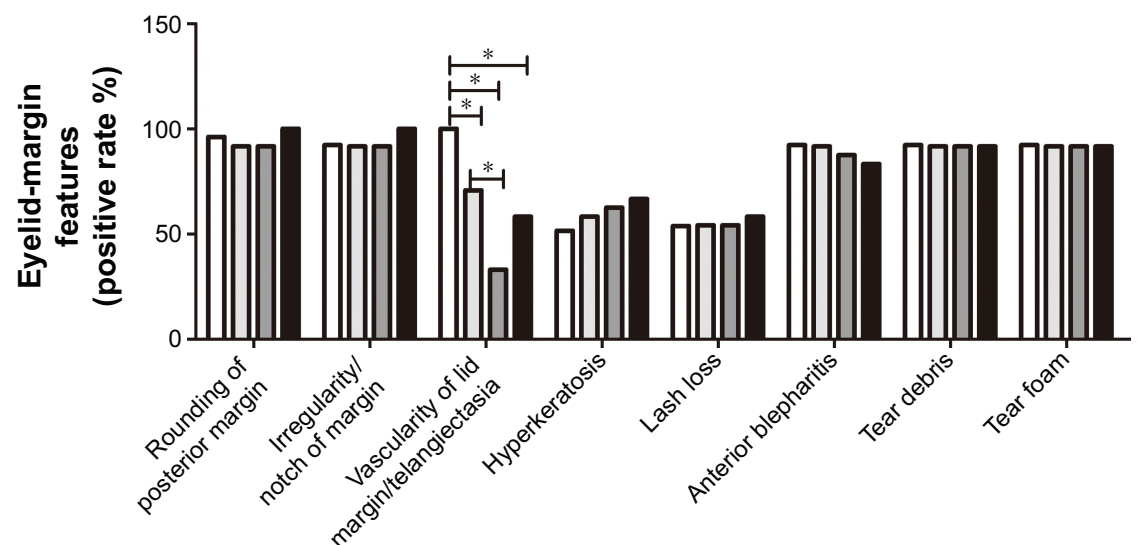

C

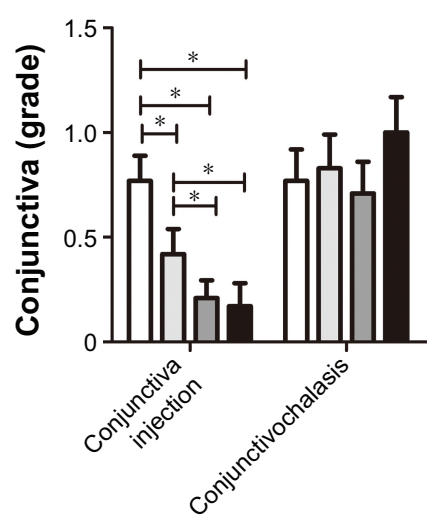

B

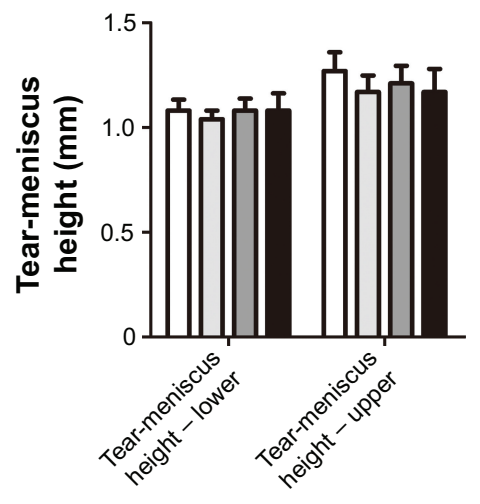

E
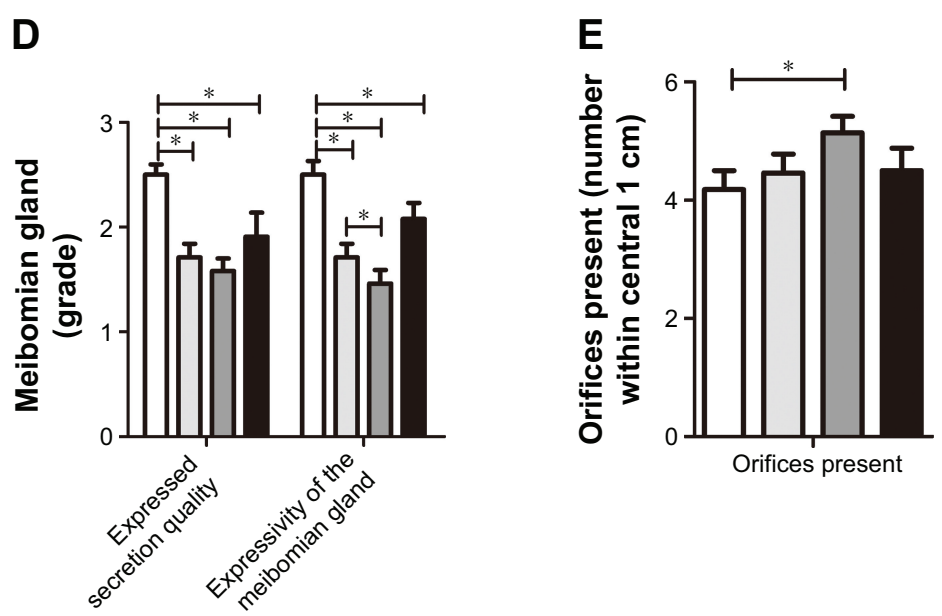

Baseline

1 week

1 month

3 months

Figure I Clinical features of meibomian gland dysfunction.

Notes: Eyelid-margin features, meibomian gland expression, tear film, and conjunctiva were graded at I day before and I week, I month, and 3 months after intra-meibomian gland injection of bevacizumab. (A) Eyelid-margin features; (B) tear-meniscus height; (C) conjunctiva injection and conjunctivochalasis; (D) expressed secretion quality and expressivity of the meibomian gland; (E) orifices within central $\mathrm{I} \mathrm{cm}$. $* P<0.05$. 
Table I Grading of clinical features and examinations before and after bevacizumab injection at I week, I month, and 3 months

\begin{tabular}{|c|c|c|c|c|}
\hline Symptoms and signs & Baseline & I week & I month & 3 months \\
\hline \multicolumn{5}{|l|}{ Eyelid margin } \\
\hline Rounding of posterior margin (\%) & 96.2 & 91.7 & 91.7 & 100 \\
\hline Irregularity/notching of margin (\%) & 92.3 & 91.7 & 91.7 & 100 \\
\hline Vascularity of lid margin/telangiectasia (\%) & 100 & $70.8^{*}$ & $33^{*, \#}$ & $58.3^{*}$ \\
\hline Hyperkeratosis (\%) & 61.5 & 58.3 & 62.5 & 66.7 \\
\hline Lash loss (\%) & 53.8 & 54.2 & 54.2 & 58.3 \\
\hline Anterior blepharitis (\%) & 92.3 & 91.7 & 87.5 & 83.3 \\
\hline \multicolumn{5}{|l|}{ Conjunctiva and tear film } \\
\hline Conjunctiva injection & $0.77(0.53-1.00)$ & $0.42(0.17-0.66)^{*}$ & $0.21(0.03-0.38)^{*, \#}$ & $0.17(-0.8 \mathrm{I} \text { to } 0.4 \mathrm{I})^{*, \#}$ \\
\hline Conjunctivochalasis & $0.77(0.46-1.08)$ & $0.83(0.5 I-1.15)$ & $0.71(0.39-1.03)$ & $1.00(0.62-1.38)$ \\
\hline Tear debris (\%) & 92.3 & 91.7 & 91.7 & 91.7 \\
\hline Tear foam $(\%)$ & 92.3 & 91.7 & 91.7 & 91.7 \\
\hline Tear-meniscus height - upper & $1.08(0.97-1.19)$ & $1.04(0.96-1.13)$ & $1.08(0.96-1.2)$ & $1.08(0.9-1.4 I)$ \\
\hline Tear-meniscus height - lower & $1.27(1.09-1.45)$ & $1.17(1.0-1.33)$ & $1.21(1.03-1.38)$ & $1.17(0.92-1.41)$ \\
\hline TBUT & $1.96(1.37-2.55)$ & $2.96(2.42-3.49)^{*}$ & $3.96(3.33-4.59)^{*, \#}$ & $3.25(1.95-4.55)^{*}$ \\
\hline \multicolumn{5}{|l|}{ Meibomian gland } \\
\hline Number present (central I cm) & $4.18(3.52-4.85)$ & $4.46(3.79-5.12)$ & $5.14(4.55-5.72)^{*}$ & $4.5(3.67-5.34)$ \\
\hline Expressed secretion quality & $2.5(2.29-2.7 I)$ & I.7I (1.44-1.97)* & $1.58(1.34-1.83)^{*}$ & $1.91(1.41-2.42)^{*}$ \\
\hline Expressivity of meibomian gland & $2.5(2.24-2.76)$ & I.7I (1.44-1.97)* & $1.46(1.18-1.74)^{*, \#}$ & $2.08(\mid .76-2.4 I)^{*}$ \\
\hline Corneal staining & $1.42(0.83-2.02)$ & $0.5(0.15-0.85)^{*}$ & $0.83(0.30-1.37)^{*}$ & $0.91(0.14-1.68)^{*}$ \\
\hline OSDI & $51.94(44.17-59.7 I)$ & $27.57(21.06-34.08)^{*}$ & $22.54(18.15-26.93)^{* \#}$ & $22.08(11.75-32.42)^{*}$ \\
\hline
\end{tabular}

Notes: $* P<0.05$ vs baseline; ${ }^{*} P<0.05$ vs I week. Data are presented as mean $(95 \% \mathrm{Cl})$ unless otherwise specified.

Abbreviations: TBUT, tear-breakup time; OSDI, Ocular Surface Disease Index.

$1.71 \pm 0.13, P<0.0001), 1$ month $(1.58 \pm 0.12, P<0.0001$; $1.46 \pm 0.13, P<0.0001)$ and 3 months $(1.91 \pm 0.23, P<0.0009$ $2.08 \pm 0.15, P=0.008)$ compared to baseline. MG expressivity improved significantly 1 month after injection when compared with that at 1 week $(P=0.014)$ and remained stable for 3 months, as shown in Figure 1D.

However, no other significant changes to eyelid-margin features were observed after bevacizumab injection. In sum, lid-margin vascularity, conjunctival injection, secretion quality, and expressivity improved significantly after bevacizumab injection, and these effects persisted for at least 3 months.

\section{Corneal staining, TBUT, and OSDI score}

Corneal staining images, TBUTs, and OSDI scores collected before and 1 week, 1 month, and 3 months after bevacizumab
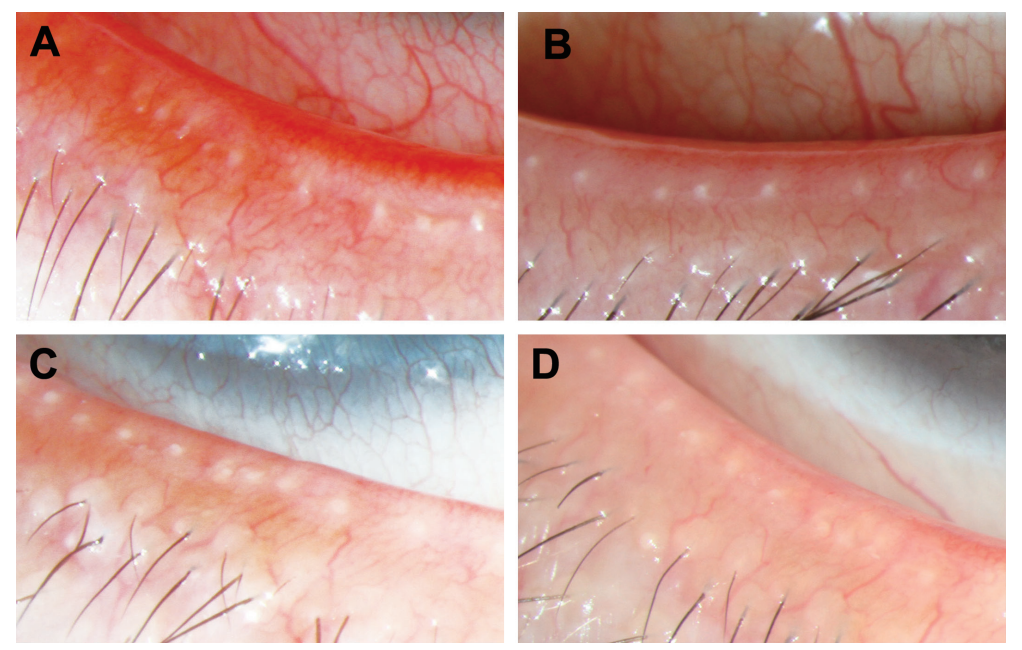

Figure 2 Eyelid-margin features.

Notes: Obtained using an IM 900 slit lamp before and after intra-meibomian gland injection of bevacizumab: (A) I day before injection; (B) I week after injection; (C) I month after injection; (D) 3 months after injection. Lid-margin vascularity decreased significantly I week postinjection (B) and continued to decrease for I month after the injection $(\mathbf{C})$ compared with the baseline $(\mathbf{A})$ and I-week postinjection time points. At 3 months (D), lid-margin vascularity sustained a relatively reduced rate compared with baseline. Similarly, the conjunctival injection was remarkably reduced after intra-meibomian gland injection compared to baseline, and the greatest reduction was observed at I month (C). 

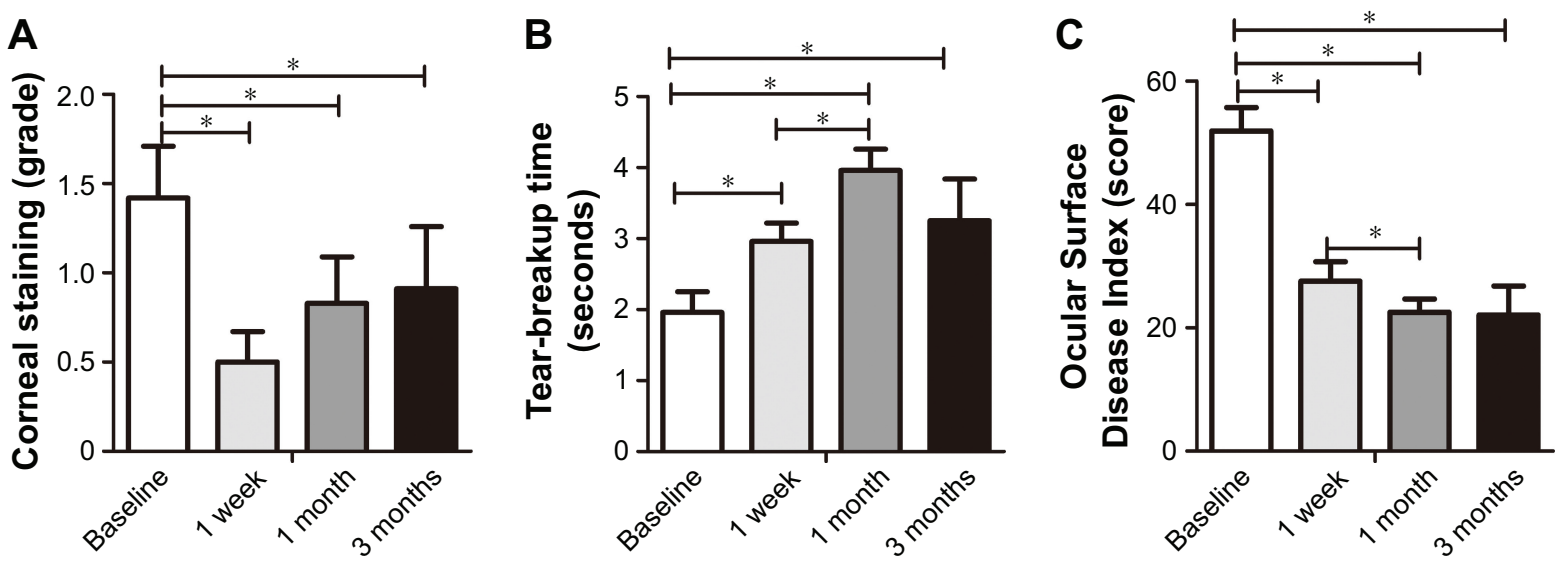

Figure 3 Corneal staining, TBUT, and OSDI score.

Notes: Results of corneal staining, TBUT, and OSDI score before injection and I week, I month, and 3 months after intra-meibomian gland injection of bevacizumab: (A) corneal staining; (B) TBUT; (C) OSDI score. Significance levels were set at $* P<0.05$.

Abbreviations: TBUT, tear-breakup time; OSDI, Ocular Surface Disease Index.

injections are presented in Figure 3 and summarized in Table 1. When compared with baseline (1.42 \pm 0.29$)$, significant improvements in corneal staining were observed at 1 week $(0.5 \pm 0.17, P=0.007), 1$ month $(0.83 \pm 0.26, P=0.009)$, and 3 months $(0.91 \pm 0.35, P=0.033)$ after injection, as shown in Figure 4. Additionally, as shown in Figure 3B, significantly longer TBUT was observed 1 week $(2.96 \pm 0.26)$ after bevacizumab injection than at the baseline $(1.96 \pm 0.29$,
$P=0.001)$, and it continued to lengthen over time. TBUT at 1 month (3.96 \pm 0.3$)$ after injection was longer than TBUT at 1 week after injection $(P=0.003)$. Similarly, significantly lower OSDI scores were obtained 1 week $(27.57 \pm 3.13$, $P<0.0001), 1$ month $(22.54 \pm 2.12, P<0.0001)$, and 3 months $(22.08 \pm 4.7, P<0.0001)$ after injection than at baseline (51.94 \pm 3.77 ); the lowest OSDI value was observed 1 month after injection, as shown in Figure 3C. In sum,
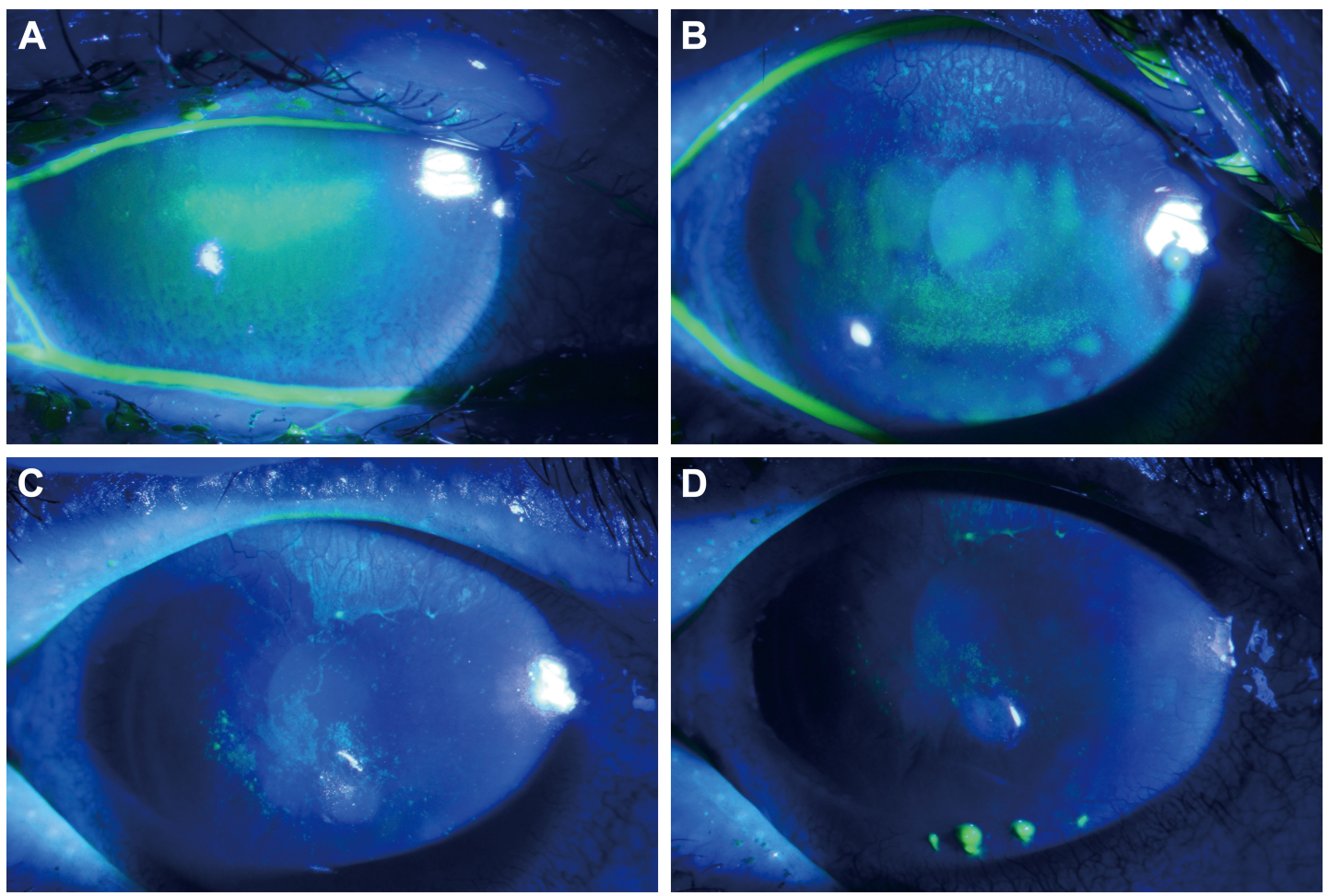

Figure 4 Corneal staining images.

Notes: Obtained using an IM 900 slit lamp and taken before and I week, I month, and 3 months after intra-meibomian gland injection of bevacizumab: (A) I day before injection; (B) I week after injection; (C) I month after injection; (D) 3 months after injection. Significant improvements were observed in images at I week (B), I month (C) and 3 months postinjection (D) compared to baseline (A). 
Table 2 Spearman's correlation analysis for sex and age associated with OSDI and TBUT improvement compared to baseline

\begin{tabular}{|c|c|c|c|c|c|c|}
\hline & \multicolumn{2}{|c|}{$\begin{array}{l}\text { Baseline- } \\
\text { I week }\end{array}$} & \multicolumn{2}{|c|}{$\begin{array}{l}\text { Baseline- } \\
\text { I month }\end{array}$} & \multicolumn{2}{|c|}{$\begin{array}{l}\text { Baseline- } \\
3 \text { months }\end{array}$} \\
\hline & $r$ & $P$-value & $r$ & $P$-value & $r$ & $P$-value \\
\hline \multicolumn{7}{|l|}{ OSDI } \\
\hline Sex & 0.12 & 0.595 & 0.308 & 0.143 & -0.207 & 0.519 \\
\hline Age & 0.03 & 0.895 & 0.343 & 0.1 & 0.488 & 0.108 \\
\hline \multicolumn{7}{|l|}{ TBUT } \\
\hline Sex & 0.027 & 0.899 & -0.033 & 0.878 & -0.767 & $0.004 *$ \\
\hline Age & 0.603 & $0.002 *$ & 0.29 & 0.169 & 0.648 & $0.023 *$ \\
\hline
\end{tabular}

Note: $* P<0.05$.

Abbreviations: TBUT, tear-breakup time; OSDI, Ocular Surface Disease Index.

corneal staining, TBUT, and OSDI improved significantly after bevacizumab injection, and the effects persisted for at least 3 months.

\section{Spearman's correlation and subgroup analysis}

The differences between the data collected before and after injection were calculated, and Spearman's correlation analysis was performed to evaluate the impact of sex and age on TBUTs and OSDI scores. Age was divided into two categories, with 60 years of age being the boundary. The results are summarized in Table 2. Age significantly influenced the improvement of TBUT at 1 week $(r=0.603$, $P=0.002)$ and 3 months $(r=0.648, P=0.023)$. Additionally, the improvement of TBUT at 3 months from baseline was impacted by sex ( $r=-0.767, P=0.004)$. However, no significant effect of age or sex on OSDI-score improvement was detected.

Thereafter, a subgroup analysis was conducted to assess the difference between sex and age on OSDI score and TBUT, as shown in Figure 5. Significantly shorter TBUT was observed in females than in males $(P<0.0001)$. Female patients tended to have improved TBUTs 1 month after injection $(P<0.0001)$, but when compared to baseline, the effect diminished 3 months after injection $(P=0.111)$. However, the TBUT of male patients improved 1 month after injection $(P=0.006)$, and the effect was sustained for 3 months $(P=0.006)$. Additionally, younger patients had longer baseline TBUTs $(P=0.032)$ and fewer adverse effects than older patients. Older patients had significant extensions to their TBUTs that were sustained during the 3 months of follow-up after injection, whereas younger patients only gained an improvement over their baseline TBUT for 1 month following injection.

All up, these data revealed that older patients had shorter baseline TBUT and that age significantly influenced the
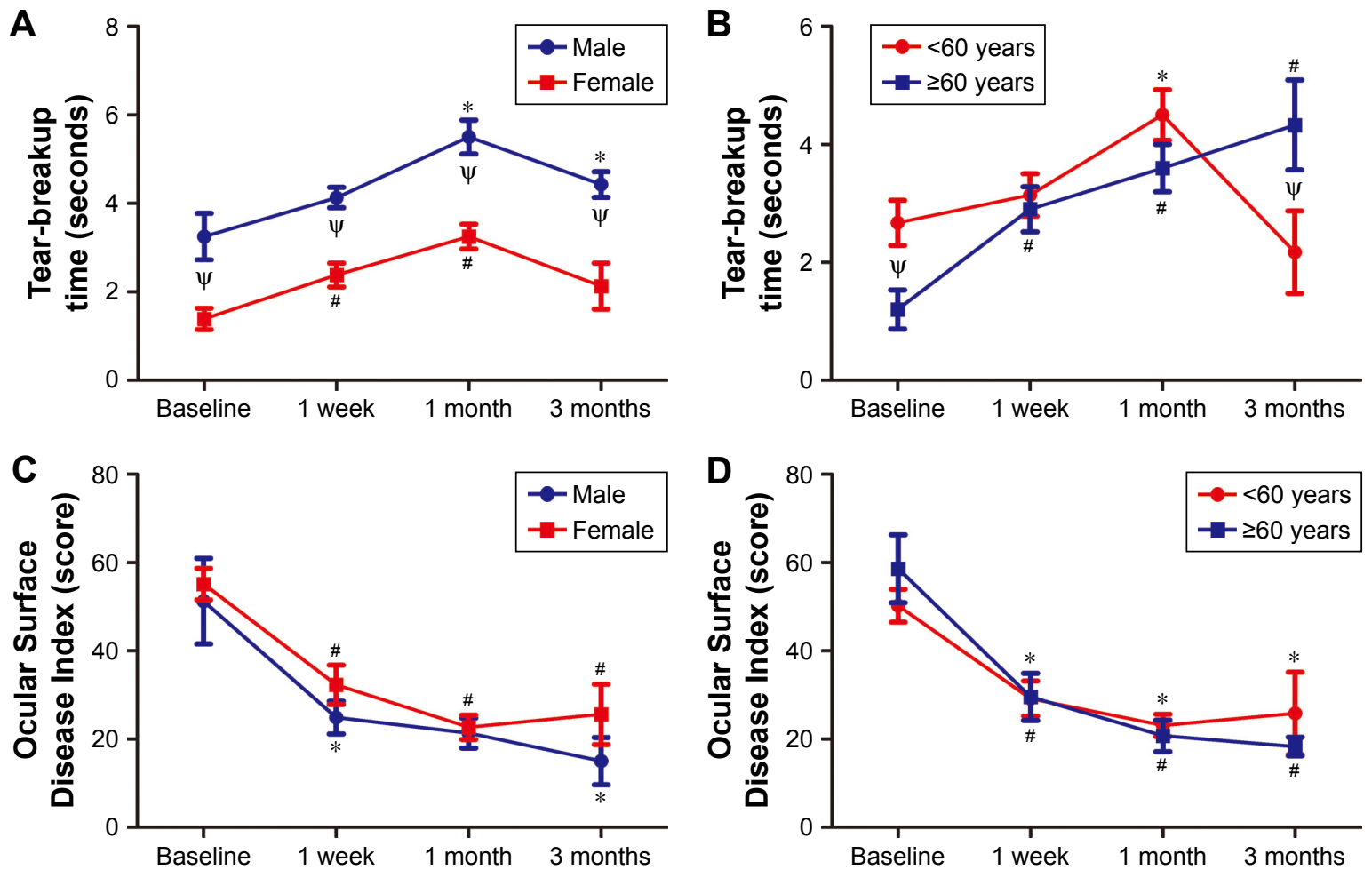

Figure 5 Subgroup analysis of TBUT and OSDI by sex and age.

Notes: Before and I week, I month, and 3 months after intra-meibomian gland injection of bevacizumab: (A) TBUT by sex; (B) TBUT by age; (C) OSDI by sex; (D) OSDI by age. ${ }^{*} P<0.05$ vs baseline for males or age $<60$ years; ${ }^{*} P<0.05$ vs baseline for females or age $\geq 60$ years; ${ }^{\psi} P<0.05$ between different sexes or ages.

Abbreviations: TBUT, tear-breakup time; OSDI, Ocular Surface Disease Index. 
extent of TBUT improvement. When compared with males, females tended to have shorter baseline TBUT and more rapid but less sustainable improvement to their TBUT.

\section{Safety data}

No obvious adverse effects were detected during any follow-up visit after intra-MG injection of bevacizumab. Best-corrected visual acuity was not affected, and the IOP was within the normal limit for all patients. A mild hemorrhage was observed at the injection spot, which was rapidly absorbed the following day. No late-onset hemorrhage or lid-margin infection occurred afterward. Systemically, blood pressure remained stable after injection.

\section{Discussion}

MGD is the main type of evaporative DE, and is characterized by a series of pathological changes, including vascularity of the lid margin and periglandular inflammation. ${ }^{1,9}$ The present study reports a novel treatment - intra-MG injection of an anti-VEGF agent - and examines its efficacy and safety in treating MGD with lid-margin vascularity.

MGD can act either as an inflammatory condition, with periglandular inflammatory pathological changes, such as meibomian keratoconjunctivitis, or a noninflammatory condition, such as in atrophic MGD. Additionally, MGD is a chronic inflammatory ocular condition that involves both eyelid margins and the $\mathrm{MG} .{ }^{28}$ Vascularity of the lid margin is a common sign of MGD that is consistent with aging, and is probably attributable to increased exposure to various insults, such as ultraviolet radiation. ${ }^{12,29}$ These insults are able to cause cell damage that triggers various pathways inducing inflammatory cytokine release ${ }^{30}$ Continuously increased stimulation from inflammatory cytokines could induce conjunctival fibroblasts to secrete VEGF, possibly causing vascularity of the ocular surface. ${ }^{31}$ Anti-VEGF has proven to be effective in treating multiple ocular problems with neovascularization, such as proliferative diabetic retinopathy and age-related macular degeneration. ${ }^{32-34}$ In our study, a 30\% reduction in lid-margin vascularity was observed 1 week after an intra-MG injection of anti-VEGF. Lid-margin vascularity continued to improve over time, with the greatest decrease in vascularity observed 1 month after injection. After 3 months, lid-margin vascularity sustained a relatively reduced rate when compared with baseline.

Additionally, a decrease in the grade of conjunctival injection was observed, which is consistent with our previous research. This result is in accordance with other studies of anti-VEGF agents on ocular neovascularization, eg, the
VEGFA trap aflibercept significantly decreased angiogenesis and ocular inflammation when compared with phosphate buffer saline and dexamethasone groups in a murine DE model. ${ }^{35}$ Bevacizumab might directly permeate the ocular surface to neutralize VEGF and prevent vascularization of the conjunctiva and lid margin. Indirectly, decreased lid-margin vascularity improves MG function, which eliminates various insults to the conjunctiva and further alleviates conjunctival injection. Generally, an intra-MG injection of bevacizumab can simultaneously reduce the vascularity of the lid margin and conjunctiva.

However, with the exception of lid-margin vascularity, the remaining eyelid margin features that were evaluated, including rounding of the posterior margin, irregularity/ notching of the margin, hyperkeratosis, lash loss, and anterior blepharitis, did not significantly improve. This result is likely attributable to the different origins of these signs in the eyelid margin. Hyperkeratosis occurred due to continuous stress on the epithelium and was encountered in atopies or nonatopic subjects, while irregularity/notching of the margin arises from the absorption of meibomian orifice tissue. ${ }^{8}$ As such, all these pathological changes are difficult to reverse solely by eliminating neovascularization. In addition, it is difficult to reverse the anatomical changes of MGD with only one intra-MG injection of bevacizumab, because the condition is formed by long-term pathological processes. Repeated injections with prolonged observation might be an effective method to remedy these symptoms.

Secretion quality and expressivity are crucial indicators used to assess MG function. ${ }^{8}$ In MGD, both secretion quality and expressivity are altered. ${ }^{36,37}$ Our research revealed that secretion quality and expressivity significantly improved 1 week, 1 month, and 3 months after intra-MG injection of the anti-VEGF agent. According to new studies on MGD, inflammation and blockage of the MG may cause stasis of the meibum inside the gland, which provides a better milieu for bacterial proliferation. ${ }^{13}$ This alteration in the ocular flora enhances the production of lipid-degrading lipases and esterases, increasing the viscosity of the meibum. ${ }^{38,39}$ Consequently, the highly viscous meibum further aggravates the blockage and inflammation of the MG, which forms a vicious self-sustaining loop. Inflammation was the target in this study, and breaking any of the links within the loop might alleviate the symptoms of MGD. Besides promoting vascular endothelial cell proliferation and migration, VEGF also acts as a factor that facilitates inflammatory processes. Directly, VEGF stimulates the release of inflammatory and chemotactic factors, such as IL6, IL8, TNF $\alpha$, and MCP1. ${ }^{18}$ 
Indirectly, VEGF can mediate the gap between vascular endothelial cells to increase vascular leakage, promoting monocyte chemotaxis and B-cell production. ${ }^{40}$ Additionally, dendritic cell-derived VEGF stimulates inflammatory angiogenesis in lymph nodes, promoting their proliferation and activation. ${ }^{41}$ Research has indicated that intraperitoneal injections of anti-VEGF agents significantly alleviate ocular surface inflammation and DE, as shown by a reduction in inflammatory cytokines and lymph nodes. ${ }^{42}$ As such, in this study, the application of an anti-VEGF agent may have disrupted the vicious cycle of MGD by obstructing the inflammatory process and preventing the release of cytokines, which improved the stasis of the meibum, as shown by the enhanced secretion quality and expressivity.

The MG secretes a mixture of multiple lipids onto the ocular surface during blinking. The secreted lipids mix with aqueous tears secreted by the lacrimal gland to form the outermost layer of the highly structured tear film. ${ }^{43}$ The lipid layer of the tear film restricts excessive evaporation of the tear film to maintain ocular surface and tear-film stability. ${ }^{44}$ During MGD, the stasis of the meibum and periglandular inflammation alter the quantity and quality of lipids secreted by the MG, which causes tear-film instability, as shown by the shortening of the TBUT. ${ }^{19,45}$ In the present study, intra-MG injections of anti-VEGF extended the TBUT during the entire 3 month follow-up period, with the longest TBUT observed 1 month after injection. Additionally, gland-secretion quality and expressivity also improved significantly, with the greatest improvements observed 1 month after injection, which follows a similar trend to what was observed for the TBUT. Additionally, tear-meniscus height did not improve significantly after injection. These results suggest that the improvement in TBUT was attributable to improved MG lipid secretion and not tear quantity. Additionally, corneal staining significantly improved after the anti-VEGF injection. The integrity of the corneal epithelium depends on the stability of the ocular surface. Previous research has revealed that intraperitoneal injection of anti-VEGF agents significantly decreases inflammatory stimuli on the ocular surface and improves corneal staining. ${ }^{42}$ Intra-MG injection of antiVEGF prolonged TBUT, which improved MG function and provided more stable tear film. In sum, the improved ocular surface with reduced inflammation and enhanced tear-film stability promoted stability of the ocular surface, which provided a better microenvironment for corneal restoration.

The OSDI is a widely used questionnaire to evaluate DE symptoms, including ocular discomfort, visual function, and environmental triggers. ${ }^{46}$ Consistently with our previous study, the present research also achieved significantly improved OSDI scores after intra-MG injection of anti-VEGF. A previous study had discussed the mechanism of VEGF in relieving neuropathic pain to eliminate the discomfort of DE. ${ }^{21,47}$ In addition to direct pain-reducing effects, anti-VEGF agents also function through an indirect mechanism to alleviate ocular discomfort. Ocular surface damage in MGD is indicated by corneal staining and pathological changes of the eyelid and $\mathrm{MG}$, all of which can cause discomfort. The improved MG function and tear-film stability caused by the anti-VEGF injection restored the ocular surface, thus relieving the discomfort.

Interestingly, we found that patients responded remarkably differently to several of the clinical signs depending on their sex and age. We calculated the differences observed before and after the injection and performed Spearman's correlation analysis to examine the impact of sex and age on OSDI and TBUT improvement. Additionally, we conducted a subgroup analysis according to sex and age to determine differences in OSDI and TBUT. Spearman's correlation analysis indicated that age significantly impacted the short-term and long-term improvement of TBUT. Taken together with the result of the subgroup analysis, older people tended to have worse baseline tear film; however, their TBUT improved after 1 week and that improvement was maintained over the following 3 months. The latest research on mice has also revealed that the DE phenotype is significantly stronger in older mice than in younger mice and that they respond differently to anti-inflammatory treatment. ${ }^{48}$ Regarding the different sexes, females tended to have significantly shorter baseline TBUTs than males, and long-term improvements in TBUT were associated with sex, as shown by our analysis. A previous population-based study determined that females were more susceptible to DED. ${ }^{49}$ Several studies have indicated that both sex hormones significantly impact $\mathrm{MG}$ function by regulating lipid production..$^{50,51}$ Androgen has a protective effect on $\mathrm{MG}$ function, ${ }^{52}$ while estrogen induces a reduction in lipid biogenesis and alters MG morphology. ${ }^{53}$ Therefore, it is reasonable that females had worse baseline and long-term improvement values on TBUT than the males in our study. Taken together, these results remind us to consider the sex and age of the patient when treating them for MGD.

In addition to efficacy, the safety of intra-MG bevacizumab injection must also be carefully considered. Upon intra-MG injection, no artifacts were introduced intraocularly or directly onto the ocular surface. As such, neither corrected visual acuity nor IOP should be impacted, a finding 
that was corroborated for all recruited patients in this study. Lid-margin hemorrhage and infection are other risk factors of invasive treatment. According to our observation, a mild hemorrhage was detected shortly after injection; however, it was absorbed rapidly by the following day. No delayed hemorrhage or lid-margin wound infection occurred thereafter. The injection itself is a strong stimulus; therefore, the systemic state and tolerance of the patient should be considered. In the present study, no obvious adverse effects were observed, and blood pressure remained stable after injection during the entire follow-up.

This pilot research is the first to explore the innovative treatment modality of intra-MG injections of the antivascularization agent bevacizumab for MGD. However, there are several limitations to this study: the small sample due to strict inclusion criteria, the short follow-up of 3 months, and the lack of a control group. Future research should focus on conducting a randomized controlled trial with larger samples and longer follow-up.

\section{Conclusion}

The present study applied a novel method - intra-MG injection of the antivascularization agent bevacizumab - as an MGD therapy, and demonstrated that the treatment is effective and safe in eliminating eyelid-margin vascularity, improving $\mathrm{MG}$ function, and relieving clinical signs and symptoms of MGD.

\section{Disclosure}

The authors report no conflicts of interest in this work.

\section{References}

1. Tsubota K, Yokoi N, Shimazaki J, et al. New perspectives on dry eye definition and diagnosis: a consensus report by the Asia Dry Eye Society. Ocul Surf. 2017;15(1):65-76.

2. Ding J, Sullivan DA. Aging and dry eye disease. Exp Gerontol. 2012; 47(7):483-490.

3. Foulks GN, Pflugfelder SC. New testing options for diagnosing and grading dry eye disease. Am J Ophthalmol. 2014;157(6):1122-1129.

4. Lemp MA, Crews LA, Bron AJ, Foulks GN, Sullivan BD. Distribution of aqueous-deficient and evaporative dry eye in a clinic-based patient cohort: a retrospective study. Cornea. 2012;31(5):472-478.

5. Bron AJ, Yokoi N, Gafney E, Tiffany JM. Predicted phenotypes of dry eye: proposed consequences of its natural history. Ocul Surf. 2009; $7(2): 78-92$

6. Mathers WD, Lane JA. Meibomian gland lipids, evaporation, and tear film stability. Adv Exp Med Biol. 1998;438:349-360.

7. McCulley JP, Shine WE. Meibomian gland function and the tear lipid layer. Ocul Surf. 2003;1(3):97-106.

8. Tomlinson A, Bron AJ, Korb DR, et al. The international workshop on meibomian gland dysfunction: report of the diagnosis subcommittee. Invest Ophthalmol Vis Sci. 2011;52(4):2006-2049.

9. Foulks GN, Bron AJ. Meibomian gland dysfunction: a clinical scheme for description, diagnosis, classification, and grading. Ocul Surf. 2003;1(3): 107-126.
10. Matsumoto Y, Shigeno Y, Sato EA, et al. The evaluation of the treatment response in obstructive meibomian gland disease by in vivo laser confocal microscopy. Graefes Arch Clin Exp Ophthalmol. 2009; 247(6):821-829.

11. Jester JV, Parfitt GJ, Brown DJ. Meibomian gland dysfunction: hyperkeratinization or atrophy? BMC Ophthalmol. 2015;15 Suppl 1:156.

12. Lin PY, Cheng CY, Hsu WM, et al. Association between symptoms and signs of dry eye among an elderly Chinese population in Taiwan: the Shihpai Eye Study. Invest Ophthalmol Vis Sci. 2005;46(5): 1593-1598.

13. Baudouin C, Messmer EM, Aragona P, et al. Revisiting the vicious circle of dry eye disease: a focus on the pathophysiology of meibomian gland dysfunction. Br J Ophthalmol. 2016;100(3):300-306.

14. Ibrahim OM, Dogru M, Matsumoto Y, et al. Oxidative stress induced age dependent meibomian gland dysfunction in $\mathrm{Cu}, \mathrm{Zn}$-superoxide dismutase-1 (Sod1) knockout mice. PLoS One. 2014;9(7):e99328.

15. Zhang SD, He JN, Niu TT, et al. Bacteriological profile of ocular surface flora in meibomian gland dysfunction. Ocul Surf. 2017;15(2): 242-247.

16. Shibuya M. Vascular Endothelial Growth Factor (VEGF) and Its Receptor (VEGFR) Signaling in Angiogenesis: A Crucial Target for Anti- and Pro-Angiogenic Therapies. Genes \& cancer. 2011;2(12):1097-1105.

17. Van Bergen T, Vandewalle E, Van de Veire S, et al. The role of different VEGF isoforms in scar formation after glaucoma filtration surgery. Experimental Eye Research. 2011;93(5):689-699.

18. Yoo SA, Kwok SK, Kim WU. Proinflammatory role of vascular endothelial growth factor in the pathogenesis of rheumatoid arthritis: prospects for therapeutic intervention. Mediators of Inflammation. 2008; 2008:129873.

19. Enriquez-de-Salamanca A, Castellanos E, Stern ME, et al. Tear cytokine and chemokine analysis and clinical correlations in evaporative-type dry eye disease. Molecular Vision. 2010;16:862-873.

20. Sandler A, Gray R, Perry MC, et al. Paclitaxel-carboplatin alone or with bevacizumab for non-small-cell lung cancer. The New England Journal of Medicine. 2006;355(24):2542-2550.

21. Jiang X, Lv H, Qiu W, Liu Z, Li X, Wang W. Efficiency and safety of subconjunctival injection of anti-VEGF agent - bevacizumab - in treating dry eye. Drug Des Devel Ther. 2015;9:3043-3050.

22. Ozcura F, Aydin S, Helvaci MR. Ocular Surface Disease Index for the diagnosis of dry eye syndrome. Ocul Immunol Inflamm. 2007;15(5): 389-393.

23. Caffery BE, Josephson JE. Corneal staining after sequential instillations of fluorescein over 30 days. Optom Visi Sci. 1991;68(6):467-469.

24. Thomas ML, Szeto VR, Gan CM, Polse KA. Sequential staining: the effects of sodium fluorescein, osmolarity, and $\mathrm{pH}$ on human corneal epithelium. Optom Visi Sci. 1997;74(4):207-210.

25. Schulze MM, Hutchings N, Simpson TL. Grading bulbar redness using cross-calibrated clinical grading scales. Invest Ophthalmol Vis Sci. 2011; 52(8):5812-5817.

26. Mimura T, Iida M, Oshima R, et al. Changes of conjunctivochalasis after cataract surgery via a superior transconjunctival sclerocorneal incision. Int Ophthalmol. 2017;37(3):691-700.

27. Pult H, Purslow C, Murphy PJ. The relationship between clinical signs and dry eye symptoms. Eye (Lond). 2011;25(4):502-510.

28. Wei Y, Asbell PA. The core mechanism of dry eye disease is inflammation. Eye Contact Lens. 2014;40(4):248-256.

29. Hykin PG, Bron AJ. Age-related morphological changes in lid margin and meibomian gland anatomy. Cornea. 1992;11(4):334-342.

30. Di Girolamo N. Signalling pathways activated by ultraviolet radiation: role in ocular and cutaneous health. Curr Pharm Des. 2010;16(12): $1358-1375$.

31. Nagineni CN, William A, Cherukuri A, Samuel W, Hooks JJ, Detrick B. Inflammatory cytokines regulate secretion of VEGF and chemokines by human conjunctival fibroblasts: role in dysfunctional tear syndrome. Cytokine. 2016;78:16-19.

32. Ba J, Peng RS, Xu D, et al. Intravitreal anti-VEGF injections for treating wet age-related macular degeneration: a systematic review and meta-analysis. Drug Des Devel Ther. 2015;9:5397-5405. 
33. Pozarowska D, Pozarowski P. The era of anti-vascular endothelial growth factor (VEGF) drugs in ophthalmology, VEGF and anti-VEGF therapy. Cent Eur J Immunol. 2016;41(3):311-316.

34. Zhang Y, Han Q, Ru Y, Bo Q, Wei RH. Anti-VEGF treatment for myopic choroid neovascularization: from molecular characterization to update on clinical application. Drug Des Devel Ther. 2015;9:3413-3421.

35. Kwon JW, Choi JA, Shin EY, et al. Effect of trapping vascular endothelial growth factor-A in a murine model of dry eye with inflammatory neovascularization. Int J Ophthalmol. 2016;9(11):1541-1548.

36. Ong BL, Larke JR. Meibomian gland dysfunction: some clinical, biochemical and physical observations. Ophthalmic Physiol Opt. 1990; 10(2):144-148.

37. Bron AJ, Benjamin L, Snibson GR. Meibomian gland disease: classification and grading of lid changes. Eye (Lond). 1991;5(Pt 4):395-411.

38. Murillo N, Aubert J, Raoult D. Microbiota of Demodex mites from rosacea patients and controls. Microb Pathog. 2014;71-72:37-40.

39. Kosik-Bogacka DI, Lanocha N, Lanocha A, et al. Role of Demodex folliculorum in the pathogenesis of blepharitis. Acta Ophthalmol. 2012; 90(7):e579.

40. Ferrara N, Gerber HP, LeCouter J. The biology of VEGF and its receptors. Nat Med. 2003;9(6):669-676.

41. Salvi V, Vermi W, Gianello V, et al. Dendritic cell-derived VEGF-A plays a role in inflammatory angiogenesis of human secondary lymphoid organs and is driven by the coordinated activation of multiple transcription factors. Oncotarget. 2016;7(26):39256-39269.

42. Goyal S, Chauhan SK, Dana R. Blockade of prolymphangiogenic vascular endothelial growth factor $\mathrm{C}$ in dry eye disease. Arch Ophthalmol. 2012;130(1):84-89.

43. Butovich IA. Lipidomics of human meibomian gland secretions: chemistry, biophysics, and physiological role of meibomian lipids. Prog Lipid Res. 2011;50(3):278-301.
44. Stahl U, Willcox M, Stapleton F. Osmolality and tear film dynamics. Clin Exp Optom. 2012;95(1):3-11.

45. Joffre C, Souchier M, Gregoire S, et al. Differences in meibomian fatty acid composition in patients with meibomian gland dysfunction and aqueous-deficient dry eye. Br J Ophthalmol. 2008;92(1):116-119.

46. Finis D, Pischel N, König C, et al. [Comparison of the OSDI and SPEED questionnaires for the evaluation of dry eye disease in clinical routine]. Ophthalmologe. 2014;111(11):1050-1056. German.

47. Lin J, Li G, Den X, et al. VEGF and its receptor-2 involved in neuropathic pain transmission mediated by $\mathrm{P} 2 \mathrm{X}_{2 / 3}$ receptor of primary sensory neurons. Brain research bulletin. 2010;83(5):284-291.

48. Steven P, Braun T, Krösser S, Gehlsen U. [Influence of aging on severity and anti-inflammatory treatment of experimental dry eye disease]. Klin Monbl Augenheilkd. 2017;234(5):662-669. German.

49. Han SB, Hyon JY, Woo SJ, Lee JJ, Kim TH, Kim KW. Prevalence of dry eye disease in an elderly Korean population. Arch Ophthalmol. 2011 129(5):633-638.

50. Schirra F, Suzuki T, Richards SM, et al. Androgen control of gene expression in the mouse meibomian gland. Invest Ophthalmol Vis Sci. 2005;46(10):3666-3675.

51. Schirra F, Richards SM, Liu M, Suzuki T, Yamagami H, Sullivan DA. Androgen regulation of lipogenic pathways in the mouse meibomian gland. Exp Eye Res. 2006;83(2):291-296.

52. Nanavaty MA, Long M, Malhotra R. Transdermal androgen patches in evaporative dry eye syndrome with androgen deficiency: a pilot study. Br J Ophthalmol. 2014;98(4):567-569.

53. Suzuki T, Schirra F, Richards SM, Jensen RV, Sullivan DA. Estrogen and progesterone control of gene expression in the mouse meibomian gland. Invest Ophthalmol Vis Sci. 2008;49(5):1797-1808.
Drug Design, Development and Therapy

\section{Publish your work in this journal}

Drug Design, Development and Therapy is an international, peerreviewed open-access journal that spans the spectrum of drug design and development through to clinical applications. Clinical outcomes, patient safety, and programs for the development and effective, safe, and sustained use of medicines are the features of the journal, which

\section{Dovepress}

has also been accepted for indexing on PubMed Central. The manuscript management system is completely online and includes a very quick and fair peer-review system, which is all easy to use. Visit http://www.dovepress.com/testimonials.php to read real quotes from published authors.

Submit your manuscript here: http://www.dovepress.com/drug-design-development-and-therapy-journal 